\title{
Orientación Educativa para la Vida Familiar como Medida de Apoyo para el Desempeño de la Parentalidad Positiva
}

\section{Educational Guidance for Families. A Support for Developing a Positive Parenting}

\author{
Raquel-Amaya Martínez González \\ Universidad de Oviedo
}

\author{
Carlos Manuel Becedóniz Vázquez \\ Observatorio de la Infancia y la Adolescencia P. de Asturias
}

\begin{abstract}
Resumen. En este capítulo se plantea la necesidad de promover la Orientación Educativa para la Vida Familiar como medida de apoyo a las familias para el desempeño de la Parentalidad Positiva, tal como sugieren las Recomendaciones del Consejo de Europa a sus Estados Miembro (2006). Se revisan con detalle estas recomendaciones y se mencionan algunas medidas que se desarrollan en España para apoyar a familias que se encuentran en situaciones especiales, haciendo alusión a los Convenios-Programa que se han venido desarrollando en los últimos años financiados entre el gobierno central y el de las Comunidades y Ciudades Autónomas. Asimismo, se mencionan otras medidas preventivas y comunitarias de apoyo socio-educativo para todas las familias a través del desarrollo de Programas de Orientación Educativa para el Ejercicio Positivo del Rol Parental y se sugieren algunas líneas de actuación que requieren el trabajo multidisciplinar, colaborativo y en red de los diversos agentes sociales y entidades que trabajan en favor de las familias.

Palabras clave: parentalidad positiva, medidas de apoyo a las familias, orientación educativa para la vida familiar.
\end{abstract}

Summary. This paper focuses on the need to promote Educational Guidance for Family Life as a means to support families in developing a Positive Parenting role, such as suggested by the Council of Europe (2006). This Recommendation is discussed in some detail and some measures that had been put into practice in Spain to support families under special circumstances are revised; in order to do so, Programme-Agreements which had been developed in the last years sponsored by both the central government and the one of the Spanish Autonomous Cities and Communities are described. Moreover, other preventive and community measures focused on socio-educative support for all families through Positive Parenting Programmes are mentioned. This paper ends with some suggestions about actions which require a multidisciplinary, collaborative net approach among the diverse social entities and institutions working in favour of families.

Key words: positive parenting, measures to support families, educative guidance for family life.

La correspondencia sobre este artículo puede dirigirse a la primera autora a través del correo electrónico: raquelamaya@gmail.com
Agradecimientos: Los autores de este documento agradecen la colaboración e información suministrada por la Dirección General de Política Social, de las Familias y la Infancia del Ministerio de Sanidad y Política Social. 


\section{Orientación Educativa para la Vida Familiar}

La Orientación Educativa para la Vida Familiar está cobrando cada vez más importancia en nuestra sociedad por la necesidad que sienten muchas familias de recibir asesoramiento para cumplir adecuadamente su rol educativo y parental con respecto a sus hijos ${ }^{2}$.

El rol socializador de la familia está universalmente aceptado en todas las sociedades por las funciones asistenciales, educativas y adaptativas que realiza tanto con los menores como con los adultos, contribuyendo a su desarrollo personal y también al desarrollo de la sociedad al generar capital social (Baumrid, 1973; Musitu, Buelga, Lila, y Cava, 2001; Pérez Díaz, Rodríguez y Sánchez, 2001). Por ello, se entiende que la familia constituye un núcleo fundamental de la sociedad. Esta función socializadora se hace especialmente relevante con los menores, a quienes se entiende que hay que proteger para garantizar los derechos propios de la Infancia (Naciones Unidas, 1959).

Los padres y madres quieren lo mejor para sus hijos, para su desarrollo y bienestar personal. Para ello, cuentan en este momento con mejores condiciones socio-económicas y de dotación de recursos y servicios culturales y sociales de los que contaron otros padres y madres en épocas pasadas. Pero, a la vez, la complejidad de factores que operan en la sociedad actual (diversidad social y personal, multiculturalidad, cada vez más sofisticadas tecnologías y medios de información y comunicación, incorporación de la mujer al mundo laboral, cambio de valores y de formas de interacción y convivencia, entre otros), unidos a los cambios de estructura que tienen lugar en muchas familias como resultado de procesos de separación, divorcio y creación de nuevas relaciones de pareja, así como la dedicación de un amplio espacio de tiempo diario por parte de los dos progenitores a funciones laborales fuera del hogar, hacen que los padres y madres perciban que educar a los menores y jóvenes en la actualidad sea un proceso más complejo de lo que lo fue en momentos anteriores.

2 Con el término genérico "hijo(s)", niño(s) se hace referencia tanto a hijos/niños varones como a hijas/niñas.
De modo, que la dotación de mayores recursos sociales y culturales del momento actual no siempre lleva asociada necesariamente una percepción de mayor seguridad en el modo y dinámicas con que los padres y madres educan a sus hijos. Especialmente en la etapa de la adolescencia incrementa el riesgo de abandono escolar, de fracaso académico, de consumo de drogas, de embarazos prematuros y de fuertes conflictos interpersonales, lo que lleva a la mayoría de los padres y madres a preguntarse ¿cómo hacer para prevenir estos problemas? Y, en caso de que aparezcan ¿cómo hacer para afrontarlos? En muchas ocasiones, los padres y madres buscan apoyo contrastando sus experiencias parentales con las de otros padres y madres o consultando al profesorado en los centros escolares. Pero no siempre, ni unos ni otros, encuentran las respuestas apropiadas.

Por ello, la Orientación Educativa para la Vida Familiar se hace cada vez más necesaria en la sociedad actual. En otros trabajos y publicaciones hemos definido y concretado los objetivos, principios y contenidos de esta disciplina (Martínez González, 1999; 2001; Martínez González, Pérez Herrero y Álvarez Blanco, 2007). Baste recordar en este momento que se trata de un servicio de apoyo a las familias, que puede contribuir, de darse las condiciones adecuadas, al desarrollo humano y personal de todos los componentes del sistema familiar, ya sean menores o adultos, en todas las etapas de su desarrollo biológico y evolutivo y en cualquier tipo de familia con independencia de su estructura, dinámica interna o de cualquier otro factor de diversidad. La Orientación Educativa para la Vida Familiar está dirigida a todas las familias, tengan o no problemáticas de convivencia interna en distintos grados. Tiene una finalidad fundamentalmente formativa, preventiva y no tanto terapéutica, y actúa desde un enfoque de intervención comunitaria. Por eso, los programas y acciones de orientación educativa familiar pueden desarrollarse tanto en centros escolares como en centros sociales, centros de salud, centros de servicios sociales o en cualquier otra entidad o institución que busque facilitar el ejercicio positivo del rol parental y el bienestar del menor y de la convivencia familiar. 
Los efectos positivos que se derivan de esta orientación no se agotan en las personas ni en las familias sobre las que actúa directamente, sino que trascienden a la sociedad, al considerarse que la familia es una estructura básica de aquella, que contribuye con sus dinámicas internas a la formación de la ciudadanía -tanto de los menores como de los adultos-. De aquí, que en el momento actual se considere que la familia no tiene solo una dimensión privada, sino también pública (Consejo de Europa, 2006) y, por tanto, deba ser atendida con apoyos y recursos sociales. Estos apoyos pueden concretarse y dirigirse, entre otros, al ámbito de la educación familiar, de la orientación y mediación, al económico, al de atención y cuidado de personas dependientes -incluyendo a los niños-, al de la conciliación de la vida laboral, personal y familiar, o al de atención a familias desfavorecidas, en situación de riesgo y con graves problemas en la dinámica familiar (abusos, violencia, malos-tratos y otras situaciones que son atendidas desde los servicios sociales).

Estos ámbitos de intervención están siendo contemplados actualmente desde la Administración Pública del Estado (Ministerio de Sanidad y Política Social, Dirección General de Política Social de las Familias y de la Infancia, http://www.msc.es/ politica Social/familiasInfancia/home.htm) con medidas de ayuda, entre otras, para dependencia, prestaciones sociales y por hijos a cargo, servicios a familias con hijos menores de tres años, familias numerosas, etc. Asimismo, se han cofinanciado con fondos estatales programas de apoyo a familias en situaciones especiales, gestionados por las Comunidades Autónomas (administraciones competentes en esta materia) a través de Convenios-Programa con dichas Comunidades: 1) Programa de Educación Familiar y Atención a Familias Desfavorecidas o en Situación de Riesgo y a Familias Monoparentales, 2) Programa de Orientación y/o Mediación Familiar y Puntos de Encuentro Familiar, y 3) Programa de Apoyo a Familias en cuyo seno se produce Violencia Familiar.

Además de estos programas dirigidos a familias en situaciones especiales, las actuales Recomendaciones Europeas que emanan del Consejo de Europa sobre Promoción de la Parentalidad Positiva
(2006) introducen la conveniencia de extender estas medidas de apoyo - sobre todo las referidas a la educación, asesoramiento y orientación familiar- a todas las familias, y no solo a las que se encuentren en situaciones de riesgo. Se trata de adoptar una perspectiva preventiva y comunitaria para promover el desarrollo de estrategias y competencias parentales que faciliten construir dinámicas positivas de convivencia en todas las familias.

\section{Recomendaciones Internacionales del Consejo de Europa para el Fomento de la Parentalidad Positiva}

La dimensión pública que adquiere la familia por contribuir a proteger a los menores, a sostener el bienestar personal y social y la formación de la ciudadanía a través del cumpliendo de sus funciones educativas, de socialización y asistenciales, ha llevado al Parlamento Europeo y al Consejo de Europa $^{3}$ a formular recomendaciones a los Estados Miembro para que estimulen medidas de apoyo a las familias. En dichas recomendaciones se indica la necesidad de conjugar acciones integradas y coordinadas de todos los sectores sociales con la finalidad de contribuir a mejorar la calidad de la convivencia familiar, a proteger los derechos de los niños, y con ello a construir un futuro próspero de la sociedad. De ahí, que el European Social Charter (ETS No. 163) formule en su artículo 16 que "la familia, como unidad básica fundamental de la sociedad, tiene derecho a recibir apoyo y protección social, legal y económica para asegurar todo su potencial de desarrollo".

Un resumen de las recomendaciones europeas mencionadas se incluyen en la tabla 1 .

Con todo, las instituciones europeas recomiendan a sus Estados Miembro4: 1) reconocer el papel fundamental que desempeña la familia en la sociedad, 2) destacar entre sus muchas funciones, su labor

\footnotetext{
${ }^{3}$ Council of Europe document (p.2): Recommendation Rec(2006)19 of the Committee of Ministers to member states on policy to support positive parenting. Adopted by the Committee of Ministers on 13 December 2006 at the 983rd meeting of the Ministers' Deputies.

4 Comunicado Final y Declaración Política de los Ministerios Europeos con competencias en Asuntos Familiares: Reunión 28, Lisboa, Portugal, 16-17 Mayo, 2006.
} 
Tabla 1. Recomendaciones de Medidas de Apoyo a las familias formuladas por el Consejo de Ministros de los Estados Miembro de la Unión Europea

Recomendaciones del Consejo de Ministros de los Estados Miembro de la Unión Europea para promover Medidas de Apoyo a las familias relacionadas con:

- Las responsabilidades parentales en la sociedad moderna y los apoyos sociales necesarios para ello (No. R (84) 4), (Recomendación de la Asamblea Parlamentaria 751 (1975)).

- La erradicación de la violencia en la familia (No. R (85) 4); (Recomendación de la Asamblea Parlamentaria 1666 (2004)).

- El desarrollo de políticas familiares coherentes e integradas (No. R (94) 14); (Recomendación de la Asamblea Parlamentaria 1074 (1988)) relacionadas con medidas materiales de apoyo a la familia: económicas, de prevención de la pobreza, conciliación de la vida familiar y laboral, etc.

- La responsabilidad de los padres y madres y del profesorado en la educación de los niños (Recomendación de la Asamblea Parlamentaria 1501 (2001)).

- La conciliación de la vida laboral y familiar (No. R (96) 5).

- El bienestar de las familias monoparentales (No. R (97) 4).

- El derecho de los niños a participar en la vida familiar y social (No. R (98) 8); (Recomendación de la Asamblea Parlamentaria 1121 (1990)).

- Los derechos de los niños alojados en residencias institucionales (Rec (2005)5).

- Los derechos y plena participación en la sociedad de las personas con discapacidad para mejorar su calidad de vida (Rec(2006)5).

- La mediación familiar y la igualdad de sexos (Recomendación de la Asamblea Parlamentaria 1639 (2003)).

Fuente: Elaboración Propia adaptada del Council of Europe Document (p.2): Recommendation Rec(2006)19 of the Committee of Ministers to Member States on Policy to Support Positive Parenting (Adopted by the Committee of Ministers on 13 December 2006 at the 983rd Meeting of the Ministers' Deputies).

socializadora y educativa con respecto al desarrollo pleno e integral de los menores y, en consecuencia, 3) crear las condiciones necesarias a nivel legislativo, administrativo, económico, etc. para apoyar a las familias con recursos económicos, de tiempo y de otro tipo, y también para promover la calidad de vida y de convivencia familiar a través del ejercicio positivo del rol parental. Todo ello con la finalidad de garantizar los derechos de formación y trato de los niños como personas y ciudadanos. Entre estos derechos se destacan el de protección, participación y expresión, así como el de ser escuchados y tenidos en cuenta.

El desempeño positivo del rol parental es definido en las Recomendaciones del Consejo de Europa 5 como "el conjunto de conductas parentales que procuran el bienestar de los niños y su desarrollo integral desde una perspectiva de cuidado, afecto, protección, enriquecimiento y seguridad personal, de no violencia, que proporciona reconocimiento personal y pautas educativas e incluye el establecimiento de límites para promover su completo desarrollo, el sentimiento de control de su propia vida y puedan alcanzar los mejores logros tanto en el ámbito familiar como académico, con los amigos y en el entorno social y comunitario" ("Positive Parenting": Refers to parental behaviour based on

5 Council of Europe Document (p.2): Recommendation Rec(2006)19 of the Committee of Ministers to Member States on Policy to Support Positive Parenting. Adopted by the Committee of Ministers on 13 December 2006 at the 983rd Meeting of the Ministers' Deputies.

Intervención Psicosocial

Vol. 18, n. 2, 2009 - Págs. 97-112 the best interests of the child that is nurturing, empowering, non-violent and provides recognition and guidance which involves setting of boundaries to enable the full development of the child.... to achieve their best at home, in school, with friends and in community).

Para ello se requiere la actuación conjunta de ambos padres, de modo que se espera que se impliquen por igual y de manera activa en la educación de sus hijos.

Entre las actuaciones parentales positivas que destacan las Recomendaciones del Consejo de Europa por sus efectos beneficiosos sobre el desarrollo de los niños, se encuentran las mencionadas en la tabla 2.

El Consejo de Europa recomienda que estas actuaciones parentales positivas sean promovidas y apoyadas institucionalmente a través de servicios y programas educativos que tengan en cuenta las necesidades específicas de las familias, tanto en lo que respecta a la consideración de los diversos ciclos evolutivos de la vida familiar y edades de los hijos, como a su diversidad: características socioeconómicas, laborales, culturales, tipología monoparental o biparental, personas con enfermedad, discapacidad, adicciones, $\mathrm{u}$ otras situaciones que presenten necesidades específicas.

Entre los servicios y acciones que se están llevando a cabo actualmente en los Estados Miembro de la Unión Europea para apoyar a las familias en el ejercicio positivo de su rol parental, cabe desta- 
Tabla 2. Actuaciones parentales positivas que destacan las Recomendaciones del Consejo de Europa por sus efectos beneficiosos sobre el desarrollo de los niños

Actuaciones parentales positivas que destacan las Recomendaciones del Consejo de Europa por sus efectos beneficiosos sobre el desarrollo de los niños

- Proporcionar afecto y apoyo

- Dedicar tiempo para interaccionar con los hijos

- Comprender las características evolutivas y de comportamiento de los hijos a una determinada edad

- Establecer límites y normas para orientar el adecuado comportamiento de los hijos y generar expectativas de que cooperarán en su cumplimiento

- Comunicarse abiertamente con los hijos, escuchar y respetar sus puntos de vista, y promover su participación en la toma de decisiones y en las dinámicas familiares

- Reaccionar ante sus comportamientos inadecuados proporcionando consecuencias y explicaciones coherentes y evitando castigos violentos o desproporcionados

Fuente: Elaboración propia adaptada del Council of Europe Document: Recommendation Rec(2006)19 of the Committee of Ministers to Member States on Policy to Support Positive Parenting (Adopted by the Committee of Ministers on 13 December 2006 at the 983rd Meeting of the Ministers' Deputies).

Tabla 3. Servicios y acciones de los Estados Miembro de la Unión Europea para apoyar a las familias en el ejercicio positivo de su rol parental

Servicios y acciones de los Estados Miembro de la Unión Europea para apoyar a las familias en el ejercicio positivo de su rol parental

1. Servicios y centros locales, que proporcionan orientación, consejo y programas educativos como medidas preventivas de alcance general para todas las familias, informando a los padres y madres sobre pautas educativas a seguir con los hijos y en la relación de pareja.

2. Lineas de Ayuda, que tienen como finalidad establecer contactos entre los padres.

3. Programas educativos para padres y madres que tienen hijos en distintas etapas evolutivas.

4. Iniciativas para promover la formación académica y el desarrollo de los niños, que pueden ser clasificadas en tres grandes categorías: 1) programas dirigidos específicamente a los niños para estimular su rendimiento académico y prevenir el abandono escolar, 2) programas dirigidos específicamente a los padres y madres para que puedan desarrollar estrategias parentales positivas que contribuyan a la mejora del rendimiento académico de sus hijos, y 3) programas dirigidos a fomentar la cooperación entre los centros académicos y las familias.

5. Servicios y programas dirigidos a colectivos de familias con situaciones y necesidades específicas o en posible situación de riesgo: inmigrantes, minorías étnicas, personas con discapacidad, familias con hijos adolescentes, familias con dificultades socio-económicas, etc.

6. Servicios de protección de los derechos de la infancia, como los proporcionados por Save the Children y otras entidades con fines similares.

Fuente: Elaboración propia adaptada de la Recommendation Rec(2006)19 of the Committee of Ministers to Member States on Policy to Support Positive Parenting. Explanatory Report.

car seis líneas de actuación ${ }^{6}$, que se resumen en la tabla 3.

Estas iniciativas europeas de apoyo a las familias para el ejercicio positivo del rol parental son recientes en muchos de los Estados Miembro de la Unión Europea y requieren, por tanto, ser incentivadas y ampliadas. En este sentido, se espera que los gobiernos estatales y autonómicos, así como las corporaciones locales, faciliten medidas que las promuevan. Entre ellas, las Recomendaciones destacan la conveniencia de organizar acciones formativas con metodologías grupales que permitan a los padres $\mathrm{y}$ madres compartir y contrastar sus experiencias parentales y apoyarse mutuamente en la adquisición de estrategias educativas que eviten, entre otras cosas, el empleo de métodos violentos con los niños.

${ }^{6}$ Recommendation $\operatorname{Rec}(2006) 19$ of the Committee of Ministers to Member States on Policy to Support Positive Parenting. Explanatory Report.
En definitiva, se trata de enfatizar la idea de que los servicios y apoyos que se ofrezcan a las familias incluyan dos principios fundamentales: 1) reducir el impacto de los factores de riesgo e incrementar la probabilidad de que los factores de protección sean efectivos, y 2) asegurar que tanto los padres y madres como los hijos sean considerados y tratados en el sistema familiar como agentes activos con capacidad para organizar sus propias vidas.

\section{Medidas de Apoyo en España a las Familias que se encuentran en Situaciones Especiales}

En España, el Ministerio competente en materia de Familias e Infancia (en los últimos años, el Ministerio de Trabajo y Asuntos Sociales, el Ministerio de Educación, Política Social y Deporte y el Ministerio de Sanidad y Política Social, 
http://www.msc.es/politicaSocial/familiasInfancia/ home.htm), viene desarrollando al menos desde 1997 Políticas de Apoyo a las Familias que se encuentran en Situaciones Especiales (Desfavorecidas y en Situación de Riesgo). Entre estas medidas se encuentran los Convenios-Programa financiados conjuntamente entre el gobierno central y el de las Comunidades y Ciudades Autónomas, que se encuentran vinculados desde $2005^{7}$ a tres líneas de acción: 1) Programa de Educación Familiar y Atención a Familias Desfavorecidas o en Situación de Riesgo y a Familias Monoparentales, 2) Programa de Orientación y/o Mediación Familiar y Puntos de Encuentro Familiar, y 3) Programa de Apoyo a Familias en cuyo seno se produce Violencia

7 Antes de 2005 se contemplaban cuatro Convenios-Programa: 1) Programa de Educación Familiar y Atención a Familias Desfavorecidas o en Situación de Riesgo, 2) Programa de Apoyo a Familias Monoparentales, 3) Programa de Orientación y/o Mediación Familiar y Puntos de Encuentro Familiar, y 4) Programa de Apoyo a Familias en cuyo seno se produce Violencia Familiar.
Familiar. Inicialmente, en 2004, se establecieron cuatro Programas, siendo uno de ellos específico para Familias Monoparentales.

La finalidad última de estos Convenios-Programa ha sido apoyar y prestar atención a familias que se encuentran en situaciones especiales, así como prevenir que estas situaciones puedan degenerar en otras de exclusión social o de desintegración familiar. Estos programas han sido complementarios con otros que pudieran estar desarrollando también los servicios sociales en cada comunidad o ciudad autónoma. Para su puesta en marcha, las Comunidades y Ciudades Autónomas han asumido la corresponsabilidad en la financiación de cada proyecto en una cuantía no inferior al 50 por ciento del coste total, incluida la aportación que pueda realizar la Corporación Local.

Más en concreto, las características de cada uno de estos Convenios-Programa se resumen en las tablas 4, 5, 6 y 7, que incluyen información de los cuatro programas iniciales.

Tabla 4. Características del Convenio-Programa para la Educación Familiar y Atención de Familias Desfavorecidas y en Situación de Riesgo

Convenio-Programa 1: Programa para la Educación Familiar y Atención de Familias Desfavorecidas y en Situación de Riesgo

\begin{tabular}{|c|c|}
\hline Objetivo & $\begin{array}{l}\text { Intervención social individualizada de carácter integral en núcleos familiares que se encuentran en situación de } \\
\text { dificultad social o riesgo. Esta intervención contempla tanto actuaciones socioeducativas y asistenciales, como } \\
\text { de inserción sociolaboral. }\end{array}$ \\
\hline $\begin{array}{l}\text { Finalidad de las actuaciones } \\
\text { socioeducativas }\end{array}$ & $\begin{array}{l}\text { Capacitar a los adultos que desempeñan roles parentales para desenvolverse adecuadamente en el autocuidado, } \\
\text { el cuidado y educación de los hijos y la atención, organización y mantenimiento del hogar. }\end{array}$ \\
\hline Criterios y características & $\begin{array}{l}\text { Los proyectos se realizan por medio de las Corporaciones Locales, y se seleccionan de común acuerdo entre el } \\
\text { Ministerio competente y cada Comunidad Autónoma según los siguientes criterios y características: } \\
\text { a) Los proyectos deben tener un carácter integral de forma que se establezca un diagnóstico individual de cada } \\
\text { núcleo familiar objeto de la intervención y una planificación de actuaciones encaminadas a la superación de } \\
\text { la situación de necesidad que presente dicho núcleo familiar. Se contemplan actuaciones en las áreas de: } \\
\text { salud, formación para el empleo, seguimiento escolar y prevención del absentismo escolar, vivienda, etc., } \\
\text { acordes con el diagnóstico realizado de cada núcleo familiar. Asimismo, se establece un sistema de indica- } \\
\text { dores que permita la valoración continuada y final de la intervención social realizada. } \\
\text { b) Las actuaciones de educación familiar se realizan preferentemente mediante visitas domiciliarias, ya que es } \\
\text { en el hogar familiar donde las conductas desadaptadas se crean y se mantienen. Estas actuaciones están a } \\
\text { cargo de profesionales especializados en Educación Social. } \\
\text { c) La intervención familiar ha de tener un carácter continuado con el fin de lograr los objetivos propuestos para } \\
\text { cada núcleo familiar; no deberán perseguirse cambios puntuales sino dotar a la familia de suficiente compe- } \\
\text { tencia para que alcance su plena autonomía en la solución de problemas de cara al futuro. } \\
\text { d) Desde la unidad responsable de la gestión del Programa en la Comunidad Autónoma, o en su caso en las } \\
\text { Corporaciones Locales, se establecen mecanismos de coordinación con los recursos sociales existentes en la } \\
\text { zona, con el fin de facilitar el carácter integral de la intervención social en los núcleos familiares. } \\
\text { e) Los proyectos deben realizarse preferentemente en zonas y barrios desfavorecidos socialmente. }\end{array}$ \\
\hline
\end{tabular}

Fuente: Adaptado de Ministerio de Trabajo y Asuntos Sociales (2004) 
Tabla 5. Características del Convenio-Programa de Apoyo a Familias Monoparentales

Convenio-Programa 2: Programa de Apoyo a Familias Monoparentales

\begin{tabular}{l|l}
\hline Objetivo & $\begin{array}{l}\text { Atender a familias que se encuentran en situación de dificultad social, encabezadas por un solo progenitor y con } \\
\text { hijos menores de } 18 \text { años. }\end{array}$ \\
\hline
\end{tabular}

Finalidad

Prestarles apoyo en el cumplimiento de sus funciones parentales y para la superación de la situación de dificultad social en la que se encuentran.

Criterios y características

Los proyectos se seleccionan de común acuerdo entre el Ministerio competente y cada Comunidad Autónoma según los siguientes criterios y características:

a) Los proyectos deben tener un carácter integral de forma que se establezca un diagnóstico individual de cada núcleo familiar objeto de la intervención y una planificación de actuaciones encaminadas a la superación de la situación de necesidad que presente dicho núcleo familiar. Se contemplan actuaciones en las áreas de: salud, formación para el empleo, seguimiento escolar y prevención del absentismo escolar, vivienda, etc.., acordes con el diagnóstico realizado de cada núcleo familiar. Asimismo, se establece un sistema de indicadores que permita la valoración continuada y final de la intervención social realizada.

b) En la intervención social que se realice y en aquellos casos que se considere necesario, se contempla la atención psicológica individualizada o grupal de las personas que encabezan las unidades familiares por la situación de estrés que puede conllevar el asumir en solitario las responsabilidades familiares.

c) Desde la unidad responsable de la gestión del Programa en la Comunidad Autónoma, o en su caso en las Corporaciones Locales, se establecen mecanismos de coordinación con los recursos sociales existentes en la zona, con el fin de facilitar el carácter integral de la intervención social en los núcleos familiares.

d) En los proyectos se contempla la potenciación de las redes de autoayuda como medio de superar las situaciones de aislamiento que pueden presentar estos núcleos familiares.

Tabla 6. Características del Convenio-Programa de Orientación y/o Mediación Familiar y Puntos de Encuentro Familiar

Convenio-Programa 3: Programa de Orientación y/o Mediación Familiar y Puntos de Encuentro Familiar

\begin{tabular}{l|l}
\hline Objetivo & El objetivo de este programa es prevenir y atender las problemáticas generadas por los procesos de conflictivi-
\end{tabular} dad familiar, por las situaciones que impliquen un riesgo de ruptura de la familia, o por las situaciones en las que la pareja haya decidido separarse, primando en todo caso el interés superior de los menores.

También se atienden las problemáticas surgidas con el cumplimiento del régimen de visitas de los hijos establecido para los supuestos de separación o divorcio de sus progenitores, bien como consecuencia de derivación judicial, bien por indicación de los Servicios Sociales o bien por propia iniciativa de los afectados.

Finalidad

El programa se instrumenta a través de la creación o mantenimiento de Servicios de Orientación y/o Mediación Familiar, así como de aquellos servicios que faciliten el régimen de visitas.

El eje principal de intervención es la familia como sistema de relaciones interpersonales entre sus integrantes y como núcleo fundamental de su desarrollo personal.

A estos efectos, se ofrece orientación psicosocial en situaciones de crisis conyugales o de conflictos intergeneracionales, con el fin de prevenir situaciones de riesgo que desemboquen en un deterioro de la convivencia familiar y/o en la desintegración familiar.

Asimismo, se promueven acciones de Mediación Familiar en casos de ruptura de la pareja y en otros supuestos de conflictividad familiar. Se emplea la técnica de resolución de conflictos a través de la ayuda de una persona imparcial (mediador familiar) que diseña un proceso para que las partes puedan dialogar, proporcionándoles habilidades que les capaciten para tomar sus propias decisiones acerca de cómo organizar su vida futura y cómo ejercer conjuntamente sus funciones y responsabilidades parentales después de la ruptura como pareja, primando en todo el proceso el interés de los menores. No se contemplan en este programa las intervenciones que deban realizarse en cumplimiento de decisiones judiciales.

Criterios y características

Los proyectos se seleccionan de común acuerdo entre el Ministerio competente y cada Comunidad Autónoma según los siguientes criterios y características:

a) Los equipamientos y la gestión de los servicios referidos deben ser preferentemente de titularidad pública. En los casos en que su titularidad corresponda a fundaciones, ONGs u otro tipo de entidades privadas sin ánimo de lucro, deberá tratarse, en todo caso, de entidades colaboradoras de la Administración Autonómica o Local. Mediante esta colaboración, se garantiza de forma explícita y documentada su utilización pública mediante acción concertada u otras fórmulas de cooperación y gestión.

b) Los servicios de orientación y/o mediación familiar han de tener un carácter multidisciplinar y estar integrados por expertos en intervención psicosocial familiar, derecho de familia y, en su caso, en mediación familiar.

c) Los servicios que atienden la problemática relacionada con el ejercicio del derecho de visita tienen, asimismo, carácter multidisciplinar y están integrados por expertos en trabajo social, apoyo psicológico y en mediación familiar.

Fuente: Adaptado de Ministerio de Trabajo y Asuntos Sociales (2004) 
Tabla 7. Características del Convenio-Programa de Apoyo a Familias en cuyo seno se produce Violencia Familiar

Convenio-Programa 4: Programa de Apoyo a Familias en cuyo seno se produce Violencia Familiar

\begin{tabular}{l|l}
\hline Objetivo & $\begin{array}{l}\text { El programa se dirige preferentemente a la intervención social integral en aquellas familias en las que se detec- } \\
\text { tan problemas de violencia, aunque se pueden contemplar también actuaciones de tipo preventivo dirigidas a } \\
\text { determinados núcleos familiares que se encuentran en situación de riesgo. } \\
\text { A estos efectos, se considera como violencia familiar todo acto u omisión de un miembro de la familia hacia } \\
\text { otro, con consecuencias negativas para éste y que tiene como resultado un daño físico, psíquico o en su des- } \\
\text { arrollo. }\end{array}$ \\
\hline Finalidad & $\begin{array}{l}\text { El programa se instrumenta mediante la creación o ampliación de Servicios de atención y tratamiento a las fami- } \\
\text { lias. También se incluye el mantenimiento de los Servicios ya financiados con cargo a este programa. }\end{array}$ \\
\hline Criterios y características & $\begin{array}{l}\text { Los proyectos se seleccionan de común acuerdo entre el Ministerio competente y cada Comunidad Autónoma } \\
\text { según los siguientes criterios y características: } \\
\text { a) Los equipamientos y la gestión de los servicios referidos deben ser preferentemente de titularidad pública. } \\
\text { En los casos en que su titularidad corresponda a fundaciones, ONGs u otro tipo de entidades privadas sin } \\
\text { ánimo de lucro, deberá tratarse, en todo caso, de entidades colaboradoras de la Administración Autonómica } \\
\text { o Local. Mediante esta colaboración, se garantiza de forma explícita y documentada su utilización pública } \\
\text { y proporcionalmente adecuada, mediante acción concertada u otras fórmulas de cooperación y gestión. }\end{array}$ \\
b) Como parte de los proyectos, pueden contemplarse cursos de formación para los profesionales que partici- \\
pan en el programa con el fin de actualizar sus conocimientos.
\end{tabular}

Fuente: Adaptado de Ministerio de Trabajo y Asuntos Sociales (2004).

Algunos datos sobre los proyectos realizados en 2008 en las distintas Comunidades y Ciudades
Autónomas a través de estos Convenios-Programa se detallan en la tabla $8^{8}$.

Tabla 8. Datos sobre los proyectos realizados en 2008 en los tres Convenios-Programa en las distintas Comunidades

y Ciudades Autónomas españolas

\begin{tabular}{|c|c|c|c|c|c|c|c|c|}
\hline \multirow[t]{2}{*}{ CC-Ciud. AA } & \multicolumn{2}{|c|}{$\begin{array}{c}\text { Programa } 1 \\
\text { Educación Familiar }\end{array}$} & \multicolumn{2}{|c|}{$\begin{array}{c}\text { Programa } 2 \\
\text { Orientación y/o } \\
\text { Mediación y Puntos } \\
\text { de Encuentro }\end{array}$} & \multicolumn{2}{|c|}{$\begin{array}{c}\text { Programa } 3 \\
\text { Apoyo a Familias con } \\
\text { Violencia Familiar }\end{array}$} & \multicolumn{2}{|c|}{ Totales } \\
\hline & $\mathbf{N}^{0}$ Proyectos & $\mathbf{N}^{0}$ Familias & $\mathrm{N}^{0}$ Proyectos & $\mathbf{N}^{0}$ Familias & $\mathbf{N}^{0}$ Proyectos & $\mathbf{N}^{0}$ Familias & Proyectos & Familias \\
\hline Andalucía & 5 & 660 & 16 & 1.210 & 2 & 500 & 23 & 2.370 \\
\hline Aragón & 1 & 120 & 1 & 200 & 1 & 60 & 3 & 380 \\
\hline Asturias & 11 & 737 & 4 & 160 & 1 & 49 & 16 & 946 \\
\hline Baleares & 28 & 2.274 & 3 & 512 & 1 & 35 & 32 & 2.821 \\
\hline Canarias & 31 & 2.573 & 3 & 487 & 4 & 357 & 38 & 3.417 \\
\hline Cantabria & 2 & 302 & 1 & 120 & 1 & 300 & 4 & 722 \\
\hline Castilla-León & 16 & 837 & 5 & 390 & 4 & 251 & 25 & 1.478 \\
\hline Cast-Mancha & 19 & 960 & 6 & 2.365 & 7 & 487 & 32 & 3.812 \\
\hline Cataluña & 41 & 11.128 & 2 & 6.220 & 3 & 12.840 & 46 & 30.188 \\
\hline Extremadura & 29 & 1.100 & 8 & 574 & 1 & 159 & 38 & 1.833 \\
\hline Galicia & 4 & 6.982 & 11 & 1.988 & 4 & 200 & 19 & 9.170 \\
\hline Madrid & 3 & 1.580 & 2 & 1.055 & 2 & 690 & 7 & 3.325 \\
\hline Murcia & 17 & 730 & 8 & 560 & 11 & 285 & 36 & 1.575 \\
\hline La Rioja & 9 & 305 & 1 & 390 & 1 & 30 & 11 & 725 \\
\hline Valencia & 51 & 9.110 & 8 & 1.923 & 7 & 1.233 & 66 & 12.266 \\
\hline Ceuta & 1 & 60 & 1 & 25 & 1 & 1.500 & 3 & 1.585 \\
\hline Melilla & 1 & 80 & 2 & 32 & 1 & 18 & 4 & 130 \\
\hline TOTALES & 269 & 39.538 & 82 & 18.211 & 52 & 18.994 & 403 & 76.743 \\
\hline
\end{tabular}

Fuente: Adaptado de Ministerio de Sanidad y Política Social (2009).

${ }^{8}$ Los autores de este documento agradecen la colaboración e información suministrada por la Dirección General de Política Social, de las Familias y la Infancia del Ministerio de Sanidad y Política Social. 
Tabla 9. Datos sobre los proyectos realizados en 2008 en la Comunidad Autónoma del Principado de Asturias sobre el Programa-Convenio Educación Familiar y Atención de Familias Desfavorecidas y en Situación de Riesgo y de Familias Monoparentales

\begin{tabular}{|c|c|c|c|}
\hline \multicolumn{4}{|c|}{$\begin{array}{l}\text { Comunidad Autónoma del Principado de Asturias, } 2008 \\
\text { CONVENIO-PROGRAMA 1: EDUCACIÓN FAMILIAR Y ATENCIÓN DE FAMILIAS DESFAVORECIDAS Y EN SITUACIÓN } \\
\text { DE RIESGO Y DE FAMILIAS MONOPARENTALES }\end{array}$} \\
\hline $\mathrm{N}^{0}$ proyecto & Denominación del proyecto & $\begin{array}{c}\text { Titularidad/ } \\
\text { Entidad gestora }\end{array}$ & $\begin{array}{l}N^{0} \text { de familias } \\
\text { beneficiarias }\end{array}$ \\
\hline 1 & E.I.T.A.F ${ }^{9}$. de Langreo & Ayto de Langreo & 61 \\
\hline 2 & E.I.T.A.F. de Siero & Ayto de Siero & 55 \\
\hline 3 & E.I.T.A.F. de Mieres & Ayto de Mieres & 93 \\
\hline 4 & E.I.T.A.F. de Gijón & Ayto de Gijón & 96 \\
\hline 5 & E.I.T.A.F. de Oviedo & Ayto de Langreo & 90 \\
\hline \multirow[t]{2}{*}{6} & E.I.T.A.F. de la Mancomunidad Cinco Villas & & \\
\hline & y Ayuntamientos de Castrillón, Corvera y Gozon & Ayto de Pravia & 21 \\
\hline 7 & E.I.T.A.F. de Avilés & Ayto de Avilés & 92 \\
\hline 8 & E.I.T.A.F. de Laviana & Ayto de Laviana & 84 \\
\hline 9 & E.I.T.A.F. de San Martín del Rey Aurelio & Ayto de San Matín del Rey Aurelio & 70 \\
\hline 10 & E.I.T.A.F. de la Agrupación de Municipios de: Llanera, & & \\
\hline & Riosa, Morcín y Rivera de Arriba & Ayto de Llanera & 10 \\
\hline 11 & E.I.T.A.F. de la Mancomunidad del Oriente de Asturias & Ayto de Parres & 65 \\
\hline & & & 737 \\
\hline
\end{tabular}

Fuente: Adaptado de Ministerio de Sanidad y Política Social (2009).

Como se puede apreciar en la tabla 8 , el número global de proyectos desarrollados en 2008 fue de 403, con un total de 76.743 familias atendidas. El análisis por Programas indica que el dedicado a Educación Familiar y Atención de Familias Desfavorecidas y en Situación de Riesgo y Familias Monoparentales acumula el mayor número de proyectos (269), con un total de familias atendidas de 39.538 entre todas las Comunidades y Ciudades Autónomas.

A modo de ejemplo de cómo se desarrollan estos Convenios-Programa, se incluyen datos en la tabla 9 de proyectos realizados en 2008 en la Comunidad Autónoma del Principado de Asturias.

Como se puede apreciar en la tabla 9, los proyectos realizados en la Comunidad Autónoma del Principado de Asturias con relación al programa Educación Familiar y Atención de Familias Desfavorecidas y en Situación de Riesgo y de Familias Monoparentales han dado cobertura a 737

\footnotetext{
${ }^{9}$ EITAF-Equipos de Intervención Técnica de Apoyo a la Familia
}

familias a través de la actuación de los Equipos de Intervención Técnica de Apoyo a la Familia $\left(\right.$ EITAF $\left.^{10}\right)$ del Principado de Asturias.

Por lo que respecta al Convenio-Programa sobre Orientación y/o Mediación Familiar y Puntos de Encuentro Familiar, la tabla 10 muestra que durante el año 2008 se han realizado en el Principado de Asturias cuatro proyectos a cargo de distintas asociaciones colaboradoras con la administración autonómica o local, de los que han resultado beneficiadas 160 familias.

Por último, el Convenio-Programa de Apoyo a Familias en cuyo seno se produce Violencia Familiar suscrito en 2008 con la Comunidad Autónoma del Principado de Asturias, ha beneficiado a 49 familias que han participado en un proyecto llevado a cabo por Cruz Roja Española de Asturias (tabla 11).

\footnotetext{
${ }_{10}$ Para más información sobre el funcionamiento de los EITAF del Principado de Asturias consultar Becedóniz Vázquez, C. (Coord.) (2009). Los Equipos de Intervención Técnica de Apoyo a la Familia (EITAF) en el Principado de Asturias: Manual de Actuación en las Intervenciones Orientadas a la Capacitación Parental, Principado de Asturias. Observatorio de la Infancia y la Adolescencia. (www.princast.es/observainfancia).
} 
Tabla 10. Datos sobre los proyectos realizados en 2008 en la Comunidad Autónoma del Principado de Asturias sobre el Programa-Convenio Programa de Orientación y/o Mediación Familiar y Puntos de Encuentro Familiar

Comunidad Autónoma del Principado de Asturias, 2008

CONVENIO-PROGRAMA 2: PROGRAMA DE ORIENTACIÓN Y/O MEDIACIÓN FAMILIAR Y PUNTOS DE ENCUENTROFAMILIAR

\begin{tabular}{lccc}
\hline \multicolumn{1}{c}{ Denominación del proyecto } & Titularidad / Entidad gestora & Municipio & $\begin{array}{c}\mathbf{N}^{\mathbf{0}} \text { de familias } \\
\text { beneficiarias }\end{array}$ \\
\hline Programa de Orientación y Mediación Familiar en Asturias ADHOC & Asociación Adhoc & Oviedo & 60 \\
Programa de Orientación y Mediación Familiar en Asturias Defamilia & Asociación Defamilia & Oviedo & 20 \\
Programa de Puntos de Encuentro Familiar en Asturias Gijón & Asociación CAVASYM & Gijón & 25 \\
Programa de Puntos de Encuentro Familiar en Asturias Oviedo & Asociación Alternativa para el Menor & Oviedo & 55 \\
\hline Total & & $\mathbf{1 6 0}$ \\
\hline Fuente: Adaptado del Ministerio de Sanidad y Política Social (2009). & & \\
\hline
\end{tabular}

Tabla 11. Datos sobre los proyectos realizados en 2008 en la Comunidad Autónoma del Principado de Asturias sobre el Programa-Convenio Programa de Apoyo a Familias en cuyo seno se produce Violencia Familiar

\begin{tabular}{|c|c|c|c|}
\hline \multicolumn{4}{|c|}{$\begin{array}{l}\text { Comunidad Autónoma de Asturias, } 2008 \\
\text { CONVENIO-PROGRAMA 3: PROGRAMA DE APOYO A FAMILIAS EN CUYO SENO SE PRODUCE VIOLENCIA FAMILIAR }\end{array}$} \\
\hline Denominación del proyecto & Titularidad / Entidad gestora & Municipio & $\begin{array}{l}\mathrm{N}^{0} \text { de familias } \\
\text { beneficiarias }\end{array}$ \\
\hline $\begin{array}{l}\text { Programa de atención integral a familias monoparentales } \\
\text { con grave problemática sociofamiliar }\end{array}$ & Cruz Roja Española de Asturias & Oviedo & 49 \\
\hline
\end{tabular}

\section{Medidas Preventivas y Comunitarias de Apoyo \\ Socio-Educativo a las Familias a través de Programas de Orientación para el Ejercicio Positivo del Rol Parental}

Los Convenios-Programa descritos anteriormente tienen como finalidad fundamental atender y apoyar las dinámicas de familias que se encuentran en situaciones especiales a través de acciones vinculadas mayoritariamente a servicios sociales. Por su parte, el apoyo socio-educativo familiar asociado a programas de orientación para el ejercicio positivo del rol parental está dirigido a todo tipo de familias, tengan o no situaciones especiales, con independencia de su tipología, estructura, dinámica interna o ciclo vital. Se trata de actuaciones dirigidas tanto a familias normalizadas como a familias en situaciones especiales -desfavorecidas o en riesgo-, con un enfoque fundamentalmente preventivo y comunitario y basado, entre otras, en dos estrategias fundamentales: 1) fomentar los puntos fuertes y las potencialidades de las familias y de las personas, evitando centrase solo en sus limitaciones y carencias (McKnight, Kretzmann, 1990) y 2) promover redes sociales de apoyo comunitario y vecinal, dado que parte de una perspectiva de intervención comunitaria con base en la prevención primaria.

Con esta línea de actuación comunitaria y de prevención primaria se intenta dar respuesta a las necesidades de asesoramiento educativo manifestadas por padres y madres en diversos contextos profesionales y de interacción (centros escolares, centros de salud-pediatría, centros sociales, etc.) e identificadas también a través de estudios de investigación (Bornstein, 2002; Carrobles y Pérez Pareja, 2001; Lozano, 2003; Maganto y Bartau, 2004; Máiquez, Rodrigo, Capote y Vermaes, 2000; Martínez González, 1994, 1998; Martínez González y Pérez Herrero, 2004; Martínez González, Pérez Herrero y Álvarez Blanco, 2007). Asimismo, como se ha mencionado, las Recomendaciones del Parlamento Europeo y del Consejo de Europa (2006) indican 
también la conveniencia de diseñar, desarrollar y evaluar programas educativos para padres y madres que tienen hijos en distintas etapas evolutivas (ver tabla 3).

La finalidad de estos programas educativos es asesorar a las familias sobre contenidos y estrategias que les permitan desarrollar competencias personales y parentales necesarias para afrontar los retos cotidianos de la vida familiar. En términos generales, las medidas existentes incluyen acciones dirigidas a (Martínez-González, 2001; MartínezGonzález, 2007; Martínez González y Rodríguez Ruiz, 2007): 1) mejorar el conocimiento de los padres y madres sobre las características evolutivas de sus hijos, 2) desarrollar estrategias sobre estilos educativos positivos (educación familiar) (Pourtois,1989; Durning, 1995), y 3) facilitar afrontar las dificultades y conflictos cotidianos que se producen en el seno familiar, con especial mención a nivel europeo a la prevención y control del castigo físico corporal.

Esta finalidad y efectos esperados de los programas de asesoramiento para el ejercicio positivo del rol parental los convierten en interesantes herramientas educativas para fomentar tanto el desarrollo personal de los padres, madres e hijos, como una adecuada convivencia familiar, y el comportamiento ciudadano de los menores y de los adultos. Son programas que ayudan a las familias a entender mejor su función socializadora con respecto a los hijos, y contribuyen, por tanto, a que los padres y madres se sientan más competentes para cumplir con la expectativa de generar capital social y de educar a sus hijos como ciudadanos responsables, respetuosos con las normas y demandas sociales, y cooperativos en el esfuerzo común de promover el desarrollo social.
En España, diversos autores e instituciones han aportado ideas y recursos para trabajar en esta línea. Entre ellos cabe destacar las iniciativas de Bartau, Maganto, Etxeberría y Martínez González (1999), Brunet y Negro (1994), Carrobles y Pérez Pareja (2001), Maganto y Bartau (2004), Máiquez, Rodrigo, Capote y Vermaes (2000), Martínez González (1998, 1999), Martínez González y Pérez Herrero (2004); Martínez González, Pérez Herrero y Álvarez Blanco (2007), Martínez González y Rodríguez Ruiz (2007), Merino Rodríguez et al. (1997); Radio Ecca -Canarias-, Preescolar na Casa -Galicia-, etc. Si bien, como se pone de manifiesto en las Recomendaciones del Consejo de Europa (2006) y en el Informe ChildONEurope (2007), hasta el momento esta medida de apoyo educativo se ha fomentado escasamente y requiere de un mayor análisis y desarrollo.

A modo de ejemplo, cabe citar el trabajo realizado por Martínez González (2009), quien ha elaborado un Programa-Guía para el Desarrollo de Competencias Emocionales, Educativas y Parentales donde se abordan diversas dimensiones de contenido que puede ser adaptado a las distintas etapas evolutivas de la familia según la edad de los hijos. Estas dimensiones se recogen en la tabla 12 .

El desarrollo de estas dimensiones de contenido lleva asociado un conjunto de estrategias transversales básicas para el desarrollo de valores y competencias emocionales tanto en los padres y madres como en los hijos, cuya relación se expone en la tabla 13. Estas estrategias se consideran básicas y transversales porque permiten a los padres y madres orientar sus acciones en diversos campos de actuación de interés tanto para las familias como para la

Tabla 12. Dimensiones de Contenido del Programa-Guía para el Desarrollo de Competencias Emocionales, Educativas y Parentales

Dimensiones de Contenido del Programa-Guía para el Desarrollo de Competencias Emocionales, Educativas y Parentales (Martínez González, 2009)

1. Información sobre las características evolutivas de las personas en una determinada etapa evolutiva

2. Habilidades cognitivas, de relajación y de autorregulación emocional

3. Autoestima y asertividad en el desarrollo de la función educativa y parental

4. Emociones y Comunicación: Hábitos y habilidades de escucha y de expresión verbal, gestual y paraverbal

5. Estrategias de negociación y de resolución positiva de conflictos

6. Habilidades para establecer límites y regulación del comportamiento en los hijos

Fuente: Martínez González (2009). 
sociedad, por ejemplo: fomento de la corresponsabilidad doméstica y de la educación de los hijos, desarrollo de hábitos de alimentación saludables, prevención del consumo de drogas, fomento de la lectura y de los hábitos de estudio, prevención del abandono y del fracaso escolar, utilización adecuada del tiempo libre, etc. programas y servicios que promueven la parentalidad positiva en varios países de la Unión Europea se encuentra su escasa disponibilidad, bien sea porque no se ofertan en número suficiente, porque no se desarrollan en todas las áreas geográficas, o porque no pueden acoger a todas las familias interesadas en participar, dada su metodología participativa, o bien,

Tabla 13. Relación entre Contenidos, Estrategias, Valores y Competencias que promueve el Programa-Guía para el Desarrollo de Competencias Emocionales, Educativas y Parentales

\begin{tabular}{|c|c|c|}
\hline \multicolumn{3}{|c|}{$\begin{array}{c}\text { Relación entre Contenidos, Estrategias, Valores y Competencias que promueve el Programa-Guía para el Desarrollo de Competencias } \\
\text { Emocionales, Educativas y Parentales (Martínez González, 2009) }\end{array}$} \\
\hline Dimensiones de Contenido & Estrategias & Valores y Competencias \\
\hline ETAPA DE DESARROLLO DE LA PERSONA & Ajuste de Expectativas & - Respeto mutuo \\
\hline \multirow{2}{*}{$\begin{array}{l}\text { NECESIDAD DE ATENCIÓN, AFECTO, } \\
\text { RESPETO Y RECONOCIMIENTO }\end{array}$} & Comportamiento Cooperativo, No conflictivo & $\begin{array}{l}\text { - Cooperación } \\
\text { - Responsabilidad }\end{array}$ \\
\hline & Comportamiento No Cooperativo, Conflictivo & $\begin{array}{l}\text { - Autorregulación emocional } \\
\text { - Relajación } \\
\text { - Tolerancia a la frustración }\end{array}$ \\
\hline AUTO-ESTIMA Y ASERTIVIDAD & Estimulación & $\begin{array}{l}\text { - Confianza y seguridad en uno mismo } \\
\text { - Tolerancia a la frustración }\end{array}$ \\
\hline $\begin{array}{l}\text { ESCUCHAR Y EXPRESAR } \\
\text { SENTIMIENTOS Y OPINIONES }\end{array}$ & $\begin{array}{l}\text { Escucha activa } \\
\text { Mensajes YO }\end{array}$ & - Respeto Mutuo \\
\hline RESOLUCIÓN DE PROBLEMAS & $\begin{array}{l}\text { - Explorar alternativas } \\
\text { - Acuerdos y Negociación }\end{array}$ & $\begin{array}{l}\text { - Resolución de problemas } \\
\text { - Toma de decisiones } \\
\text { - Asumir consecuencias } \\
\text { - Responsabilidad }\end{array}$ \\
\hline $\begin{array}{c}\text { LÍMITES Y REGULACIÓN DEL } \\
\text { COMPORTAMIENTO } \\
\text { CONSTRUCCIÓN DE LA DISCIPLINA }\end{array}$ & $\begin{array}{l}\text { - Premios y Castigos } \\
\text { - Consecuencias Coherentes } \\
\text { - Persistencia }\end{array}$ & $\begin{array}{l}\text { - Asumir consecuencias } \\
\text { - Responsabilidad }\end{array}$ \\
\hline
\end{tabular}

Fuente: Martínez González (2009).

La metodología de actuación que se propone para el desarrollo de este Programa-Guía es cooperativa entre iguales, experiencial, participativa, y basada en dinámicas de grupo -tal como proponen las Recomendaciones del Consejo de Europa- coordinados por un profesional experto en orientación e intervención educativa para la vida familiar.

Esta metodología grupal puede reconducirse hacia otra de carácter individual cuando se requiera trabajar con familias que presenten necesidades específicas o que estén en situación de riesgo social y precisen una intervención más personalizada.

Entre las limitaciones que se han descrito de los

Intervención Psicosocial

Vol. 18, n. ㄹ 2, 2009 - Págs. 97-112 en algunos casos, por el coste de su implementación (ChilONEurope, 2007).

Una segunda limitación es que estas escasas actuaciones y servicios se ofrecen fundamentalmente a familias que se encuentran en situación de riesgo social, y no a todas las familias en general, quienes también necesitan asesoramiento y apoyo educativo. Asimismo, es una limitación la escasa coordinación que suele haber entre las diferentes entidades implicadas en la atención a las familias. Una limitación más es la escasa participación de los padres varones en estos programas de formación (Martínez González y Pérez Herrero, 2004). 


\section{Líneas Necesarias de Actuación para Apoyar a las Familias en el Ejercicio Positivo del Rol Parental}

Los aspectos analizados hasta el momento llevan a plantear la necesidad de promover diversas líneas de actuación para apoyar a las familias en el ejercicio positivo del rol parental. Entre ellas cabe destacar: 1) fomentar la Orientación e Intervención Educativa para la Vida Familiar tanto desde una perspectiva aplicada, como académica y de investigación, 2) estimular las relaciones de colaboración entre las distintas entidades que trabajan en conexión con las familias (organismos competentes en materia de educación, salud, servicios sociales, empresas, asociaciones, fundaciones, ONGs, etc.), 3) promover la Mediación Familiar, 4) dinamizar la formación de profesionales especializados en Orientación e Intervención Educativa para la Vida Familiar y en Mediación Familiar que sepan trabajar desde una perspectiva preventiva, comunitaria, ecológica y multidisciplinar (García Villaluenga y Bolaños Cartujo, 2006), y 5) promover la investigación y la evaluación de Programas de Orientación Educativa Familiar.

La mayoría de los países de la Unión Europea muestran interés en promover la formación y la investigación para garantizar un apoyo adecuado y efectivo a las familias; por ejemplo, en el Reino Unido existe la Academia Nacional de Profesionales en el ámbito Familiar (National Academy for Parenting Practitioners-NAPP).

En España, a nivel estatal, el Observatorio de la Infancia adscrito al Ministerio competente en materia de Familias e Infancia ha promovido, entre otros, un estudio de investigación sobre Estrategias para prevenir y afrontar conflictos en las relaciones familiares (padres e hijos) con el objetivo de identificar las necesidades de asesoramiento y apoyo educativo que tienen las familias (Martínez González, Pérez Herrero y Álvarez Blanco, 2007, www.mepsyd.es/ observatorioinfancia/productos). En este estudio se analizan seis dimensiones sobre habilidades parentales relacionadas con las expresadas en las Recomendaciones del Consejo de Europa (2006). Asimismo, el Observatorio de la Infancia ha publicado el Programa-Guía para el Desarrollo de
Competencias Emocionales, Educativas y Parentales al que nos hemos referido anteriormente (Martínez González, 2009), dirigido a profesionales de la orientación educativa familiar con el fin de facilitar promover en los padres y madres el desarrollo de las competencias mencionadas y el ejercicio positivo del rol parental.

Desde el ámbito de las Comunidades Autónomas, cabe mencionar a modo de ejemplo, el Observatorio de la Infancia y la Adolescencia del Principado de Asturias, adscrito al Instituto Asturiano de Atención Social a la Infancia, Familias y Adolescencia (Consejería de Bienestar Social del Gobierno del Principado de Asturias), desde el que se ha desarrollado un Plan Integral de Infancia, Familias y Adolescencia del Principado de Asturias, que puede consultarse en http://www.graficosweb.com/observatorio. Este Observatorio está promoviendo desde el año 2007 la formación de profesionales especializados en Orientación Educativa para la Vida Familiar y la aplicación con familias usuarias de servicios sociales del Programa-Guía para el Desarrollo de Competencias Emocionales, Educativas y Parentales (2009) publicado por el Ministerio mencionado.

\section{Conclusión}

A modo de conclusión, cabe insistir en la necesidad de revisar y resaltar la función educativa, de socialización y de protección que desempeña la familia, tanto con los menores como con los adultos, y ponerla en relación con su potencialidad para generar capital humano y social y para contribuir al desarrollo social. Desde este marco se entiende la dimensión pública que tiene la familia y el énfasis de las Recomendaciones del Consejo de Europa a los Estados Miembro para apoyar a todas las familias con independencia de sus diversas circunstancias, para que puedan desarrollar adecuada y positivamente su rol parental. Esto lleva a considerar la conveniencia de introducir medidas de apoyo a las familias desde una perspectiva educativa, preventiva, comunitaria, ecológica, multidisciplinar, colaborativa y en red, que requiere la implicación y coordinación de diversos agentes sociales y entidades 
locales, autonómicas, estatales e internacionales, públicas y privadas.

Estas medidas educativas de apoyo a las familias permiten introducir la Orientación Educativa para la Vida Familiar como marco disciplinar que facilita a los adultos con responsabilidades educativas familiares desarrollar a través de acciones y programas formativos, las competencias emocionales, educativas y parentales necesarias para fomentar una adecuada convivencia familiar que contribuya al des- arrollo personal integral tanto de los menores como de los adultos.

El desarrollo de estas medidas educativas de apoyo a las familias requiere aún de normativas específicas que faciliten efectuar más análisis, dedicación, coordinación e inversión en lo que atañe a investigación, diseño y evaluación de programas y formación especializada en Orientación Educativa para la Vida Familiar, tal como se indica en el modelo de actuación del gráfico 1 (Martínez González, 2007, 2008 a,b,c).

APOYO DE INSTITUCIONES SOCIALES: ESTATALES, REGIONALES, LOCALES

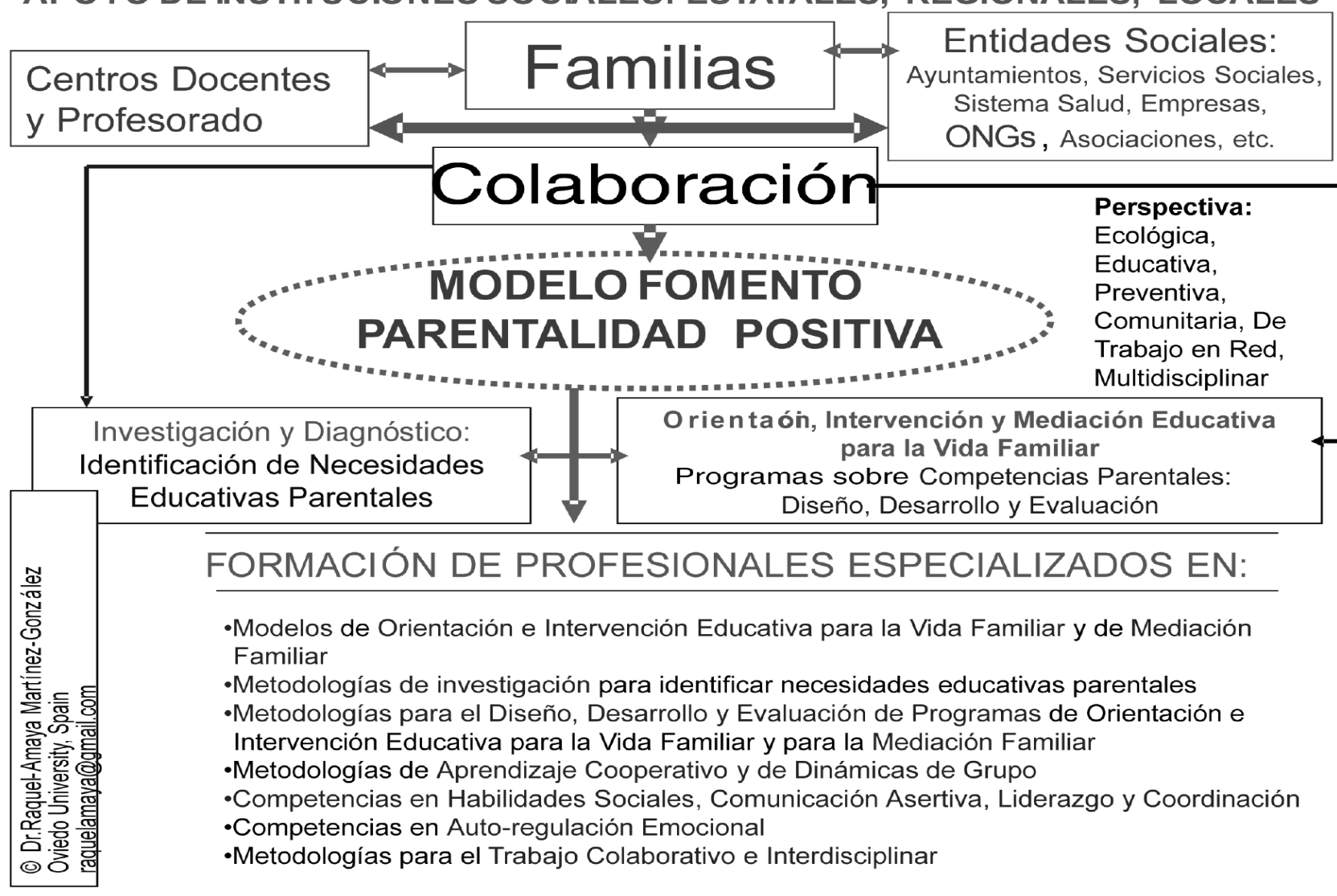

Fuente: Elaboración propia (Martínez González, 2007, 2008 a,b,c).

\section{Referencias}

Bartau, I.; Maganto, J. M.; Etxeberría, J. y Martínez González, R. A. (1999). La implicación educativa de los padres: un programa de formación, Revista Española de Orientación y Psicopedagogía. 10 (17) 43-52.

Baumrid, D. (1973). The development of instrumental competence through socialization, en A.D.
Pick (De.), Minnesota Symposium on Child Psychology. 7. Minneapolis: University of Minnesota Press.

Becedóniz Vázquez, C. M. (coord.) (2009). Los Equipos de Intervención Técnica de Apoyo a la Familia (EITAF) en el Principado de Asturias: Manual de Actuación en las Intervenciones Orientadas a la Capacitación Parental. Principado de Asturias. Observatorio de la Infancia y 
la Adolescencia (www.princest.es/observainfancia).

Bornstein, M. H. (Ed.) (2002). Handbook of Parenting: Volume 1. Children and parenting. Mahwah, NJ: Lawrence Erlbaum.

Brunet, J. J. y Negro, J. L. (1994). ¿Cómo organizar una escuela de padres? Madrid: Ediciones "San Pío X".

Carrobles, J. A. y Pérez Pareja, J. (2001). Escuela de Padres. Guía práctica para evitar problemas de conducta y mejorar el desarrollo infantil. Madrid: Pirámide.

ChildONEurope Secretariat (European Network of National Observatories on Childhood) (2007). Survey on the role of parents and the support from the Goverments in the European Union. Florence, Istituto degli Innocenti. Report delivered under the Portuguese Presidency of the European Union.

Consejo de Europa (2006). Recommendation Rec(2006)19 of the Committee of Ministers to member states on policy to support positive parenting. Explanatory Report. Estrasburgo: Consejo de Europa.

Durning, P. (1995). Education Familiale. Acteurs, processus, enjeux. París: PUF.

García Villaluenga, L. y Bolaños Cartujo, I. (2006). La Mediación familiar: una aproximación interdisciplinar. Gijón: Ediciones Trea.

Lozano, A. (2003). Factores personales, familiares y académicos que afectan al fracaso escolar en la educación Secundaria, Revista Electrónica de Investigación Psicoeducativa y Psicopedagógica, 1 (1) 43-66.

Maganto, J. M. y Bartau, I. (2004). Corresponsabilidad Familiar (COFAMI): Cómo fomentar la cooperación y la responsabilidad de los hijos. Madrid: Pirámide.

Máiquez, M. L., Rodrigo, M. J., Capote, C. y Vermaes, I. (2000). Aprender en la vida cotidiana. Un programa experiencial para padres. Madrid: Visor.

Martínez González, R. A. (1994). Familia y educación formal. Implicación de la familia en el proceso de enseñanza-aprendizaje, en Centro de Investigación, Documentación y Evaluación, Premios Nacionales de Investigación e Innova- ción Educativa 1992. Madrid: Ministerio de Educación y Ciencia, 539-561.

Martínez González, R. A. (1998). The challenge of parenting education: New demands for Spanish schools. Childhood Education.Infancy through early adolescence. International Perspectives on School-Family-Community Partnership. International Focus Issue. 74 (6) 351-354.

Martínez González, R. A. (1999). Orientación Educativa para la Vida Familiar, Revista Española de Orientación y Psicopedagogía, 10 (17) 115-127.

Martínez González, R. A. (2001). Family Education and implications for partnership with schools in Spain. En F. Smit, K. van der Wolf y P. Sleegers (Ed.). A bridge to the future. Collaboration between Parents, Schools and Communities. The Netherlands: Institute for Applied Social Sciences. University of Nijmegen. 25-32.

Martínez González, R. A. y Pérez Herrero, H. (2004). Evaluación e intervención educativa en el campo familiar. Revista Española de Orientación y Psicopedagogía, 15 (1), 89-104.

Martínez González, R. A., Pérez Herrero, M. H. y Álvarez Blanco, L. (2007). Estrategias para prevenir y afrontar conflictos en las relaciones familiares (padres e hijos). Madrid: Ministerio de Trabajo y Asuntos Sociales. Colección Observatorio de Infancia.

Martínez González, R. A. y Rodríguez Ruiz, B. (2007). Assessing parents' satisfaction with their parental role for a more effective partnerships between families and schools, en H. Phtiaka \& S. Symeonidou (Ed.) (2007). Schools and families in partnership: Looking into the future. Nicosia, Cyprus: University of Cyprus. 33-43.

Martínez González, R. A. (2007). Reunión de Expertos sobre Parentalidad Positiva. Consejo de Europa, Estrasburgo. 6-7 Diciembre. Palais de l'Europe, Salle 2.

Martínez González, R. A. (2008a). Educación Emocional y en Valores. Ponencia presentada en la Jornada sobre Familia, Escuela y Sociedad: El Reto de la Convivencia, organizada por el Observatorio de la Infancia, Ministerio de Trabajo y Asuntos Sociales y Ministerio de Educación y Ciencia, Madrid, 14 de Febrero. 
Martínez González, R. A. (2008b). Educación para la Convivencia desde el Ámbito Familiar. Ponencia presentada en el XIV Congreso Nacional y III Iberoamericano de Pedagogía: Educación, Ciudadanía y Convivencia. Diversidad y Sentido Social de la Educación. Sección: Familia, Sociedad y Redes de Comunicación. Zaragoza, 1720 Septiembre.

Martínez González, R. A. (2008c). Parentalidad Positiva: Necesidades de Asesoramiento Familiar y Programas para el Desarrollo de Competencias Parentales. Ponencia presentada en el Seminario Ministerial Hispano-Luso sobre Parentalidad Positiva: Novas Abordagens das Políticas e Medidas no âmbito da Parentalidade Positiva. Braga (Portugal), 12 de Noviembre.

Martínez González, R. A. (2009). Programa-Guía para el Desarrollo de Competencias Emocionales, Educativas y Parentales. Madrid: Minis- terio de Sanidad y Política Social.

Merino Rodríguez, C. et al. (1997). El taller de padres como recurso educativo. Granada: Ayuntamiento de Santa Fe.

McKnight, J. L. y Kretzmann, J. (1990). Mapping community capacity. Evanston: Center for Urban Affairs and Policy Research, Northwestern University.

Musitu, G., Buelga, S., Lila, M. y Cava, M. J. (2001). Familia y adolescencia. Madrid: Síntesis.

Naciones Unidas (1959). Declaración de los Derechos del Niño, aprobada por la Asamblea General de las Naciones Unidas el 20 de noviembre de 1959.

Pérez Díaz, V., Rodríguez, J. C. y Sánchez, L. (2001). La familia española ante la educación de los hijos. Barcelona: Fundación La Caixa.

Pourtois, J.P. (Ed.) (1989) Les thématiques en éducation familiale. Belgium: De Boeck Université.

Manuscrito recibido: 25/10/2009

Revisión recibida: 06/11/2009

Manuscrito aceptado: 10/11/2009 